\title{
Human Papillomaviruses and Cervical Cancer Karl Münger*‡
}

Address: The Channing Laboratory, Brigham and Women's Hospital and Department of Medicine, Harvard Medical School, 181 Longwood Avenue, Boston, MA 02115, USA

Email: Karl Münger* - karl_munger@hms.harvard.edu

* Corresponding author ‡Presenting author

from 2005 International Meeting of The Institute of Human Virology

Baltimore, USA, 29 August - 2 September 2005

Published: 8 December 2005

Retrovirology 2005, 2(Suppl I):S48 doi:I0.I I86/1742-4690-2-SI-S48

Infections with high-risk human papillomaviruses (HPVs) are associated with the vast majority of cervical carcinoma. A fraction of other anogenital tract malignancies such as penile cancer in males and vulvovaginal cancers in females as well as anal carcinomas in immunosuppressed patients and some oropharyngeal carcinomas are also associated with high-risk HPV infections. These cancers generally arise as a consequence of a molecular accident whereby a small genomic high-risk HPV fragment is irreversibly integrated into a host cell chromosome resulting in dysregulated expression of the HPV E6 and E7 oncogenes. Expression of HPV E6/E7 in epithelial cells recapitulates key steps of cervical neoplasia and cancer, which allowed for the creation of tissue culture and animal models of cervical cancer. High-risk HPV E6 and E7 proteins target important cellular growth regulatory circuits among them the p53 and retinoblastoma tumor suppressors, respectively. In addition, E6/E7 expression is a driving force for malignant progression through induction of genomic instability. Hence, highrisk HPVs are the first-ever identified, necessary and molecularly defined causative agents of a major human cancer. 